\title{
MIMO-Based Forward-Looking SAR Imaging Algorithm and Simulation
}

\author{
Ziqiang Meng, Yachao Li, Shengqi Zhu, Yinghui Quan, Mengdao Xing, and Zheng Bao \\ National Laboratory of Radar Signal Processing, Xidian University, Xi'an 710071, China \\ Correspondence should be addressed to Yachao Li; ycli@mail.xidian.edu.cn
}

Received 25 March 2014; Revised 17 June 2014; Accepted 29 June 2014; Published 15 July 2014

Academic Editor: Wei Xu

Copyright (C) 2014 Ziqiang Meng et al. This is an open access article distributed under the Creative Commons Attribution License, which permits unrestricted use, distribution, and reproduction in any medium, provided the original work is properly cited.

\begin{abstract}
Multiple-input multiple-output (MIMO) radar imaging can provide higher resolution and better sensitivity and thus can be applied to targets detection, recognition, and tracking. Missile-borne forward-looking SAR (MFL-SAR) is a new and special MIMO radar mode. It has advantage of two-dimensional (2D) imaging capability in forward direction over monostatic missile-borne SAR and airborne SAR. However, it is difficult to obtain accurate 2D frequency spectrum of the target echo signal due to the high velocity and descending height of this platform, which brings a lot of obstacles to imaging algorithm design. Therefore, a new imaging algorithm for MFL-SAR configuration based on the method of series reversion is proposed in this paper. This imaging method can implement range compression, secondary range compression (SRC), and range cell migration correction (RCMC) effectively. Finally, some simulations of point targets and comparison results confirm the efficiency of our proposed algorithm.
\end{abstract}

\section{Introduction}

Radar imaging is an emerging technology which can provide a high resolution radar image of targets in long distance independent of weather conditions and sunlight illumination, and it is being more and more widely used in military and civil fields [1-5]. Multiple-input multiple-output (MIMO) radar is an antenna system which transmits multiple probing signals via its antennas and scattering signals are received by distributed receiving antennas [6-9]. Application of MIMO radar to radar imaging could provide higher resolution and better sensitivity and thus can be applied to detection, recognition, and tracking of targets [10-12].

We consider a new MIMO radar system, that is, missileborne multistatic synthetic aperture radar (MFL-SAR) as shown in Figure 1, in which transmitting signals are transmitted simultaneously and the reflected signals at receivers can be processed independently. This configuration can be applied to missile precision terminal guidance because of its advantages of 2D imaging ability in forward direction over monostatic SAR $[13,14]$. Missile precision terminal guidance could conduct good performance in targets detection, recognition, orientation tracking, and attacking $[15,16]$. During the moving period, transmitter and receiver cooperate with each other, and transmitter irradiates the imaging area with some squint angle, while receiver receives the target echo signals in forward-looking mode. Without loss of generality and for the convenience of description, missile-borne bistatic forwardlooking SAR (MBFL-SAR) configuration is considered in this paper.

Effective and efficient imaging in this configuration is an important technology for missile precision terminal guidance; therefore, imaging algorithm for MBFL-SAR is necessary and essential. Many investigations on SAR imaging have been published and some appealing approaches have been suggested. Range-Doppler algorithm (RDA) [17-19] and chirp scaling algorithm (CSA) [20] are conventional monostatic algorithms which are applied to those configurations that have azimuth-invariant property. For bistatic SAR imaging, some other methods have been proposed. Soumekh derived the 2D spectrum expression of bistatic SAR and proposed a new RMA for bistatic configuration through two approximations in the derivation [21-23]. But this algorithm is limited to the bistatic parallel configuration because of identical velocity vectors of transmitter and receiver. Neo et al. and Davidson et al. proposed a nonlinear CS algorithm in $[24,25]$, but it neglected space-variance of Doppler frequency in range direction and influence of secondary range 


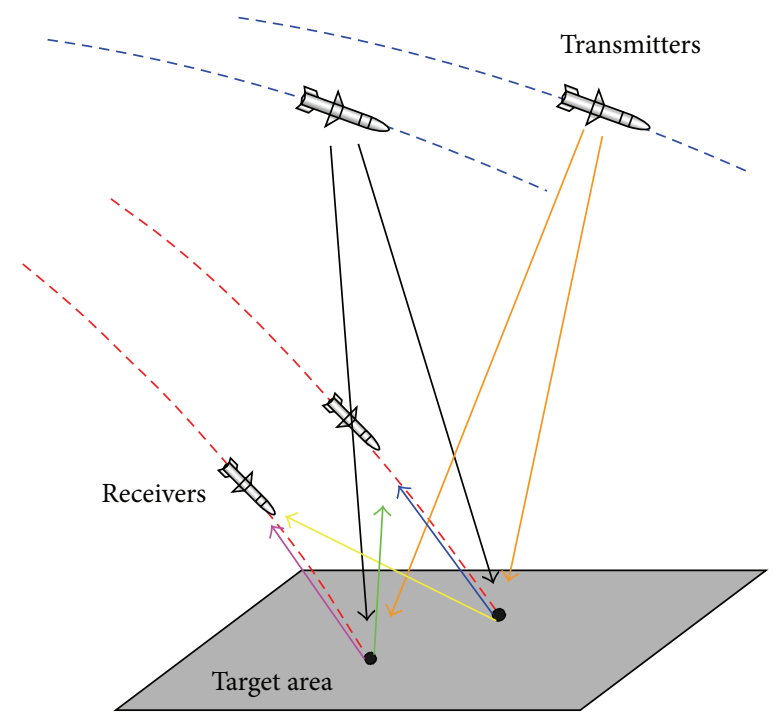

FIGURE 1: An illustration of MFL-SAR configuration.

compression (SRC) on imaging. A simple operator named "dip move out" (DMO) is used to transform a bistatic survey into a monostatic one in $[26,27]$. The initial dataset is used to convolve with the so-called smile, a short operator, and the output is the equivalent monostatic data. Unfortunately, the so-called smile is space-variant both in range direction and in azimuth direction, and the changes of wave number in range direction are also neglected.

An imaging algorithm based on the method of series reversion $[28,29]$ for MBFL-SAR is suggested in this paper. By unfolding the Doppler frequency, the stationary phase point expansion coefficients are obtained, which makes it easy to get the $2 \mathrm{D}$ frequency spectrum of the target echo signal. Then imaging method can be obtained based on the 2D frequency spectrum. Range compression is implemented in the range frequency domain, and SRC can be finished in the $2 \mathrm{D}$ frequency domain. The RCM can be corrected in the rangeDoppler domain and finally the imaging result can be got through azimuth compression.

The rest of this paper is organized as follows. Geometric configuration and signal model of MBFL-SAR are introduced in Section 2. Also, the difficulty in direct-using the principle of stationary phase (POSP) is discussed. In Section 3, the derivation of $2 \mathrm{D}$ frequency spectrum of the target echo signal is given based on the accurate approximation of the slant range history, in which the high-order terms cannot be ignored owing to the presence of high velocities and accelerations. The proposed imaging algorithm is described in Section 4 and numerical simulations are achieved to show the effectiveness of our proposed method, and the results are given in Section 5. Finally, conclusions are drawn in Section 6.

\section{Geometric Configuration and Signal Model}

Figure 2 depicts geometric configuration of MBFL-SAR, in which both transmitter and receiver travel curvilinear

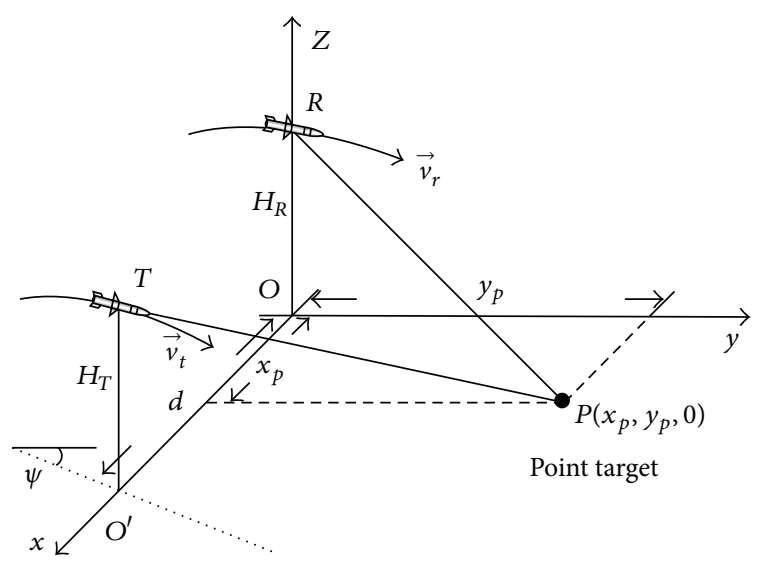

Figure 2: Geometric configuration of MBFL-SAR.

descending motion. $O$ is the origin of coordinates, $O^{\prime}$ is the projection of transmitter when the slow time $t_{m}=0$, and $\left|O O^{\prime}\right|=d$. Transmitter is moving with the original velocity vectors $\vec{v}_{t 0}=\left(v_{t x 0}, v_{t y 0}, v_{t z 0}\right)$ and acceleration vectors $\vec{a}_{t}=$ $\left(a_{t x}, a_{t y}, a_{t z}\right)$. And the ones of receiver are $\vec{v}_{r 0}=\left(0, v_{r y 0}, v_{r z 0}\right)$ and $\vec{a}_{r}=\left(0, a_{r y}, a_{r z}\right)$, respectively. There exists an included angle $\psi$ between the two planes in which the two platforms travel. The transmitter keeps illuminating the imaging area in side-looking mode, and the receiver looks in forward direction. The receiver antenna gets the echo signal reflected from the imaging area which the receiver moves towards. Assume that transmitter and receiver are at $T_{0}\left(d, 0, H_{T}\right)$ and $R_{0}\left(0,0, H_{R}\right)$ when $t_{m}=0$, and velocity vectors of them at the moment $t_{m}\left(t_{m} \neq 0\right)$ are $\vec{v}_{t}=\left(v_{t x}, v_{t y}, v_{t z}\right)$ and $\vec{v}_{r}=\left(v_{r x}\right.$, $\left.v_{r y}, v_{r z}\right)$, respectively, where $v_{t x}, v_{t y}$, and $v_{t z}$ denote the velocity components of $\vec{v}_{t}$ in the directions $x, y$, and $z$, respectively; and $v_{r x}, v_{r y}$, and $v_{r z}$ are the ones of $\vec{v}_{r}$, respectively.

The bistatic slant range at time instant $t_{m}$ can be obtained as

$$
\begin{aligned}
R_{b f}\left(t_{m}\right)= & R_{T}\left(t_{m}\right)+R_{R}\left(t_{m}\right) \\
= & \sqrt{\left(x_{t}-x_{p}\right)^{2}+\left(y_{t}-y_{p}\right)^{2}+z_{t}^{2}} \\
& +\sqrt{\left(x_{r}-x_{p}\right)^{2}+\left(y_{r}-y_{p}\right)^{2}+z_{r}^{2}},
\end{aligned}
$$

with

$$
\begin{aligned}
& x_{t}=d+v_{t x 0} t_{m}+0.5 a_{t x} t_{m}^{2}, \quad x_{r}=0, \\
& y_{t}=v_{t y 0} t_{m}+0.5 a_{t y} t_{m}^{2}, \\
& y_{r}=v_{r y 0} t_{m}+0.5 a_{r y} t_{m}^{2}, \\
& z_{t}=H_{T}+v_{t z 0} t_{m}+0.5 a_{t z} t_{m}^{2}, \\
& z_{r}=H_{R}+v_{r z 0} t_{m}+0.5 a_{r z} t_{m}^{2},
\end{aligned}
$$

where $x_{t}, y_{t}$, and $z_{t}$ represent the locations of transmitter in directions $x, y$, and $z$, respectively; and $x_{r}, y_{r}$, and $z_{r}$ are the ones of receiver, respectively. 
Suppose that the transmitted waveform is the linear frequency modulation (LFM), and scattering from $P\left(x_{p}, y_{p}, 0\right)$ to the receiver can be written as

$$
\begin{aligned}
s\left(\widehat{t}, t_{m}\right)= & w_{r}\left(\widehat{t}-\frac{R_{b f}\left(t_{m}\right)}{c}\right) w_{a}\left(t_{m}\right) \\
& \times \exp \left[j \pi \gamma\left(\widehat{t}-\frac{R_{b f}\left(t_{m}\right)}{c}\right)^{2}\right] \\
& \times \exp \left[-j \frac{2 \pi}{\lambda} R_{b f}\left(t_{m}\right)\right],
\end{aligned}
$$

where $\widehat{t}$ is the range time, $\lambda$ is the wavelength, $c$ is the speed of light, and $\gamma$ is the chirp rate. $w_{r}(\cdot)$ and $w_{a}(\cdot)$ are the range and azimuth envelopes, respectively.

By applying a range fast Fourier transform (FFT) to (3), we can obtain

$$
\begin{aligned}
s\left(f_{r}, t_{m}\right)= & W_{r}\left(f_{r}\right) w_{a}\left(t_{m}\right) \cdot \exp \left(-j \pi \frac{f_{r}^{2}}{\gamma}\right) \\
& \cdot \exp \left[-j \frac{2 \pi}{c}\left(f_{r}+f_{c}\right) R_{b f}\left(t_{m}\right)\right],
\end{aligned}
$$

where $f_{r}$ represents the range frequency.

The $2 \mathrm{D}$ frequency spectrum of the echo signal can be obtained by an azimuth FFT, expressed as

$$
S\left(f_{r}, f_{a}\right)=\int_{-\infty}^{+\infty} s\left(f_{r}, t_{m}\right) \exp \left(-j 2 \pi f_{a} t_{m}\right) d t_{m}
$$

where $f_{a}$ represents the azimuth frequency, and the phase in (5) can be written as

$$
\Theta\left(t_{m}\right)=-\pi \frac{f_{r}^{2}}{\gamma}-\frac{2 \pi}{c}\left(f_{r}+f_{c}\right) R_{b f}\left(t_{m}\right)-2 \pi f_{a} t_{m} .
$$

By applying the POSP, we have

$$
\begin{aligned}
-\frac{2 c}{f_{c}+f_{r}} f_{a}= & \frac{\alpha_{t 1}+2 \alpha_{t 2} t_{m}+3 \alpha_{t 3} t_{m}^{2}+4 \alpha_{t 4} t_{m}^{3}}{\sqrt{R_{t 0}^{2}+\alpha_{t 1} t_{m}+\alpha_{t 2} t_{m}^{2}+\alpha_{t 3} t_{m}^{3}+\alpha_{t 4} t_{m}^{4}}} \\
& +\frac{\alpha_{r 1}+2 \alpha_{r 2} t_{m}+3 \alpha_{r 3} t_{m}^{2}+4 \alpha_{r 4} t_{m}^{3}}{\sqrt{R_{r 0}^{2}+\alpha_{r 1} t_{m}+\alpha_{r 2} t_{m}^{2}+\alpha_{r 3} t_{m}^{3}+\alpha_{r 4} t_{m}^{4}}},
\end{aligned}
$$

with

$$
\begin{aligned}
& R_{t 0}=\sqrt{\left(d-x_{p}\right)^{2}+y_{p}^{2}+H_{T}^{2}}, \\
& R_{r 0}=\sqrt{x_{p}^{2}+y_{p}^{2}+H_{R}^{2}}, \\
& \alpha_{t 1}=2\left[v_{t x 0}\left(d-x_{p}\right)-y_{p} v_{t y 0}+v_{t z 0} H_{T}\right],
\end{aligned}
$$

$$
\begin{aligned}
& \alpha_{r 1}=2\left(-v_{r y 0} y_{p}+v_{r z 0} H_{R}\right), \\
& \alpha_{t 2}=v_{t x 0}^{2}+a_{t x}\left(d-x_{p}\right)+v_{t y 0}^{2}-a_{t y} y_{p}+v_{t z 0}^{2}+a_{t z} H_{T}, \\
& \alpha_{r 2}=v_{r y 0}^{2}+v_{r z 0}^{2}-a_{r y} y_{p}+a_{r z} H_{R}, \\
& \alpha_{t 3}=a_{t x} v_{t x 0}+a_{t y} v_{t y 0}+a_{t z} v_{t z 0}, \\
& \alpha_{r 3}=a_{r y} v_{r y 0}+a_{r z} v_{r z 0}, \\
& \alpha_{t 4}=\frac{1}{4}\left(a_{t x}^{2}+a_{t y}^{2}+a_{t z}^{2}\right), \\
& \alpha_{r 4}=\frac{1}{4}\left(a_{r y}^{2}+a_{r z}^{2}\right) .
\end{aligned}
$$

If $\Theta^{\prime}\left(t_{m}^{*}\right)=0$, we can get the stationary point $t_{m}^{*}$, but we can see that it is very difficult to determine the stationary point $t_{m}^{*}$ from (7), so 2D frequency spectrum of the echo signal cannot be obtained through direct POSP. Some other approaches should be taken to get $2 \mathrm{D}$ frequency spectrum of the echo signal.

\section{Derivation of 2D Frequency Spectrum Based on Series Reversion}

According to [28], it needs to take efficient approximation of the slant range $R_{b f}\left(t_{m}\right)$ before using the method of series reversion. Because of the high velocity and acceleration in the missile platform, it needs to keep the terms up to the thirdorder

$$
R_{b f}\left(t_{m}\right)=R_{b f 0}+k_{1} t_{m}+k_{2} t_{m}^{2}+k_{3} t_{m}^{3}+\cdots,
$$

with

$$
\begin{gathered}
R_{b f 0}=R_{t 0}+R_{r 0}, \\
k_{1}=\frac{\alpha_{t 1}}{2 R_{t 0}}+\frac{\alpha_{r 1}}{2 R_{r 0}}, \\
k_{2}=\frac{\alpha_{t 2}}{2 R_{t 0}}-\frac{\alpha_{t 1}^{2}}{8 R_{t 0}^{3}}+\frac{\alpha_{r 2}}{2 R_{r 0}}-\frac{\alpha_{r 1}^{2}}{8 R_{r 0}^{3}}, \\
k_{3}=\frac{\alpha_{t 3}}{2 R_{t 0}}-\frac{\alpha_{t 1} \alpha_{t 2}}{4 R_{t 0}^{3}}+\frac{\alpha_{t 1}^{3}}{16 R_{t 0}^{5}}+\frac{\alpha_{r 3}}{2 R_{r 0}}-\frac{\alpha_{r 1} \alpha_{r 2}}{4 R_{r 0}^{3}}+\frac{\alpha_{r 1}^{3}}{16 R_{r 0}^{5}} .
\end{gathered}
$$

To apply the method of series reversion, the linear range cell migration (LRCM) should be removed firstly. Let $\Theta^{\prime}\left(t_{m}\right)=0$, we have

$$
-\frac{c}{f_{c}+f_{r}} f_{a}=2 k_{2} t_{m}+3 k_{3} t_{m}^{2} .
$$

Then through the method of series reversion, the expression of the stationary phase point can be expressed by unfolded Doppler frequency as

$$
t_{m}\left(f_{a}\right)=A_{1}\left(-\frac{c}{f_{c}+f_{r}} f_{a}\right)+A_{2}\left(-\frac{c}{f_{c}+f_{r}} f_{a}\right)^{2}+\cdots,
$$

where $A_{1}=1 / 2 k_{2}, A_{2}=-3 k_{3} / 8 k_{2}^{3}$. 
Having obtained the stationary phase point, we should reintroduce the LRCM term to calculate 2D frequency spectrum of the echo signal. By (5), (6), and (12), 2D frequency spectrum of the echo signal is written as

$$
\begin{aligned}
& S_{\mathrm{MSR}}\left(f_{r}, f_{a}\right) \\
& \quad=W_{r}\left(f_{r}\right) W_{a}\left(f_{a}\right) \exp \left[-j \pi \frac{f_{r}^{2}}{\gamma}\right] \exp \left[j \Phi\left(f_{r}, f_{a}\right)\right],
\end{aligned}
$$

where

$$
\begin{aligned}
\Phi\left(f_{r}, f_{a}\right)= & -2 \pi \frac{f_{c}+f_{r}}{c} R_{b f 0}-\pi \frac{f_{r}^{2}}{\gamma} \\
& +2 \pi \frac{c}{4 k_{2}\left(f_{c}+f_{r}\right)}\left(f_{a}+\left(f_{c}+f_{r}\right) \frac{k_{1}}{c}\right)^{2} \\
& +2 \pi \frac{k_{3} c^{2}}{8 k_{2}^{3}\left(f_{c}+f_{r}\right)^{2}}\left(f_{a}+\left(f_{c}+f_{r}\right) \frac{k_{1}}{c}\right)^{3} .
\end{aligned}
$$

It can be seen that the coefficient of the third-term in the slant range is included in $\Phi\left(f_{r}, f_{a}\right)$, which indicates the presence of the high-order terms introduced by high velocity and acceleration of this configuration into 2D frequency spectrum. The imaging algorithm can be designed based on $2 \mathrm{D}$ frequency spectrum, which will be discussed in the next section.

\section{Imaging Algorithm for MBFL-SAR}

To design imaging algorithm efficiently, the phase term in (14) should be decomposed using Taylor series firstly because of the range/azimuth frequency coupling in $2 \mathrm{D}$ frequency spectrum, and the series expansions are as follows:

$$
\begin{aligned}
\Phi\left(f_{r}, f_{a}\right) \approx & \Phi_{r g}\left(f_{r}\right)+\Phi_{a}\left(f_{a}\right)+\Phi_{\mathrm{rcm}}\left(f_{r}, f_{a}\right) \\
& +\Phi_{\mathrm{src}}\left(f_{r}, f_{a}\right)+\Phi_{\text {res }}
\end{aligned}
$$

with

$$
\Phi_{r g}\left(f_{r}\right)=-\pi \frac{f_{r}^{2}}{\gamma}
$$

$$
\begin{aligned}
\Phi_{a}\left(f_{a}\right) & \\
=2 \pi\{ & \frac{1}{4 k_{2}}\left(\frac{k_{1}^{2}}{c}+2 k_{1} f_{a}+\frac{c}{f_{c}} f_{a}^{2}\right) \\
& \left.+\frac{k_{3}}{8 k_{2}^{3}}\left[\frac{k_{1}^{3}}{c}+3 k_{1}^{2} f_{a}+3 k_{1} \frac{c}{f_{c}} f_{a}^{2}+\left(\frac{c}{f_{c}}\right)^{2} f_{a}^{3}\right]\right\},
\end{aligned}
$$

$$
\begin{aligned}
\Phi_{\mathrm{rcm}}\left(f_{r}, f_{a}\right) & \\
=2 \pi f_{r}\{ & -\frac{R_{b f 0}}{c}+\frac{1}{4 k_{2}}\left[\frac{k_{1}^{2}}{c}-c\left(\frac{f_{a}}{f_{c}}\right)^{2}\right] \\
& \left.+\frac{k_{3}}{8 k_{2}^{3}}\left[\frac{k_{1}^{3}}{c}-3 k_{1} c\left(\frac{f_{a}}{f_{c}}\right)^{2}-2 c^{2}\left(\frac{f_{a}}{f_{c}}\right)^{3}\right]\right\},
\end{aligned}
$$

TABLE 1: Parameters used in the simulations.

Wavelength

Bandwidth

Sampling frequency

Pulse duration

Scene center location

Fringe point location

$\psi$

PRF

$H_{T}$

$H_{R}$

$\vec{v}_{t 0}$

$\vec{a}_{t}$

$\vec{v}_{r 0}$

$\vec{a}_{r}$

$$
\begin{aligned}
& \Phi_{\text {src }}\left(f_{r}, f_{a}\right) \\
&=2 \pi\left\{\frac{1}{4 k_{2}} \frac{c}{f_{c}}\left(\left(\frac{f_{r}}{f_{c}}\right)^{2}-\left(\frac{f_{r}}{f_{c}}\right)^{3}\right) f_{a}^{2}\right. \\
&+\frac{k_{3}}{8 k_{2}^{3}}\left[3 k_{1} \frac{c}{f_{c}}\left(\left(\frac{f_{r}}{f_{c}}\right)^{2}-\left(\frac{f_{r}}{f_{c}}\right)^{3}\right) f_{a}^{2}\right. \\
&\left.\left.+\left(\frac{c}{f_{c}}\right)^{2}\left(3\left(\frac{f_{r}}{f_{c}}\right)^{2}-4\left(\frac{f_{r}}{f_{c}}\right)^{3}\right) f_{a}^{3}\right]\right\},
\end{aligned}
$$

$$
\Phi_{\text {res }}=-2 \pi f_{c} \frac{R_{b f 0}}{c} .
$$

The term in (16) represents the range compression term which is independent of the azimuth frequency $f_{a}$. Thus the data can be range-compressed in the range frequency azimuth time domain or in the 2D frequency domain alternatively. The term in (17) denotes the azimuth compression term, dependent only on $f_{a}$ and used in the azimuth compression. It should be implemented after range compression and RCMC in the range time azimuth frequency domain as $k$-coefficients are range variant. The term in (18) indicates the RCM term which is linearly dependent on the range frequency $f_{r}$. It needs to be compensated because of the coupling between range and azimuth. Similar to the azimuth compression term, this term should be implemented in the range time azimuth frequency domain. Note that $-2 \pi\left(R_{b f 0} / c\right) f_{r}$ is the linear phase representing the location of target point; the energy of the target will be focused within the corresponding range cell after RCMC. It should be paid attention to in the imaging process. The term in (19) is the SRC term which indicates the 
TABLE 2: Image quality parameters using the proposed method.

\begin{tabular}{|c|c|c|c|c|c|c|}
\hline \multirow{2}{*}{ Parameter } & \multicolumn{2}{|c|}{ Theoretical one } & \multicolumn{2}{|c|}{ Center point } & \multicolumn{2}{|c|}{ Fringe point } \\
\hline & Azimuth & Range & Azimuth & Range & Azimuth & Range \\
\hline PSLR (dB) & \multicolumn{2}{|c|}{-13.26} & -13.26 & -13.27 & -13.15 & -13.25 \\
\hline ISLR (dB) & \multicolumn{2}{|c|}{-9.8} & -10.07 & -9.97 & -10.04 & -9.97 \\
\hline
\end{tabular}

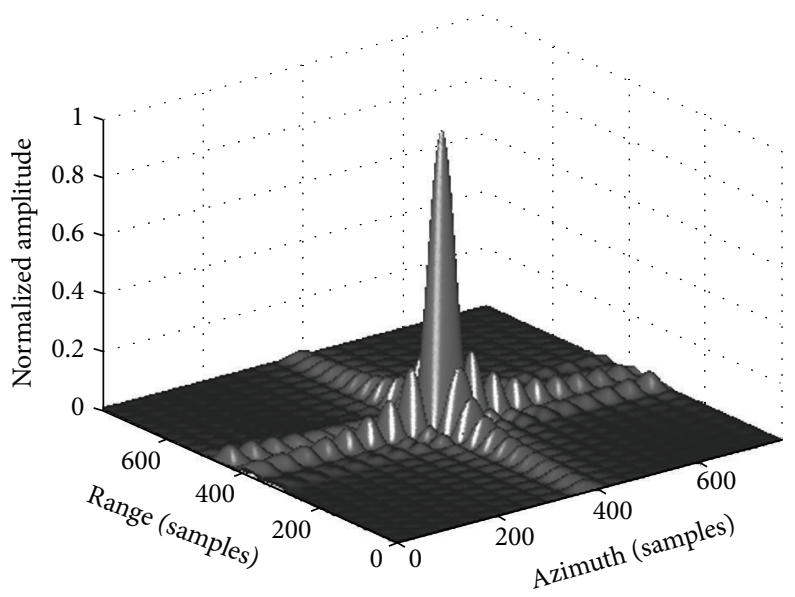

(a) 2D impulse response

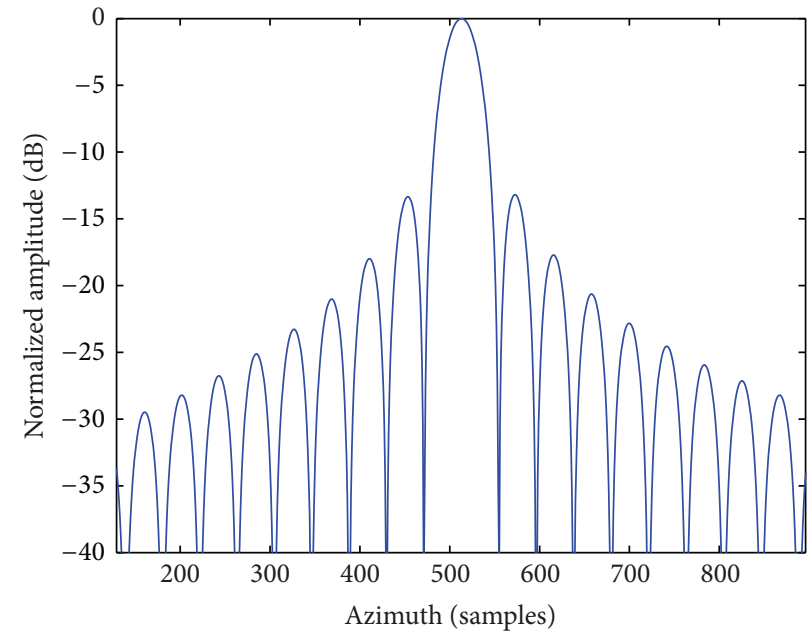

(b) Azimuth impulse response

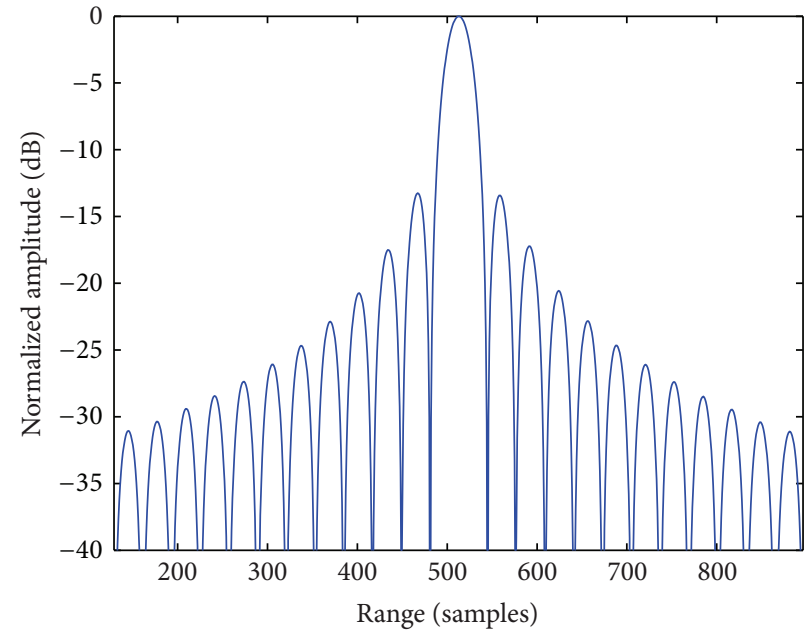

(c) Range impulse response

FIGURE 3: Center point results using the proposed range model.

coupling between $f_{r}$ and $f_{a}$. It may cause significant degradation in the imaging resolution if uncompensated and is compensated in the 2D frequency domain. The last residual term in (20) is independent of the range frequency or the azimuth frequency. It has no effect on the imaging focus and thus can be neglected in MBFL-SAR configuration.

Consequently, the main procedure of the proposed algorithm follows the following steps.

(1) Use 2D FFT to transform the echo signal to $2 \mathrm{D}$ frequency domain as shown in (13).

(2) Design the match filtering function in the 2D frequency domain to compensate the range compression term, the SRC term, and the residual term. The match filtering function $H_{1}\left(f_{r}, f_{a}\right)$ can be obtained as follows:

$$
H_{1}\left(f_{r}, f_{a}\right)=\exp \left[-j\left(\Phi_{r g}\left(f_{r}\right)+\Phi_{\text {src }}\left(f_{r}, f_{a}\right)+\Phi_{\text {res }}\right)\right] \text {. }
$$

(3) Apply range inverse fast Fourier transform (IFFT) to convert the signal to the range time azimuth frequency domain and correct the RCM term.

(4) Finish azimuth compression by multiplying the range time azimuth frequency domain signal with the 


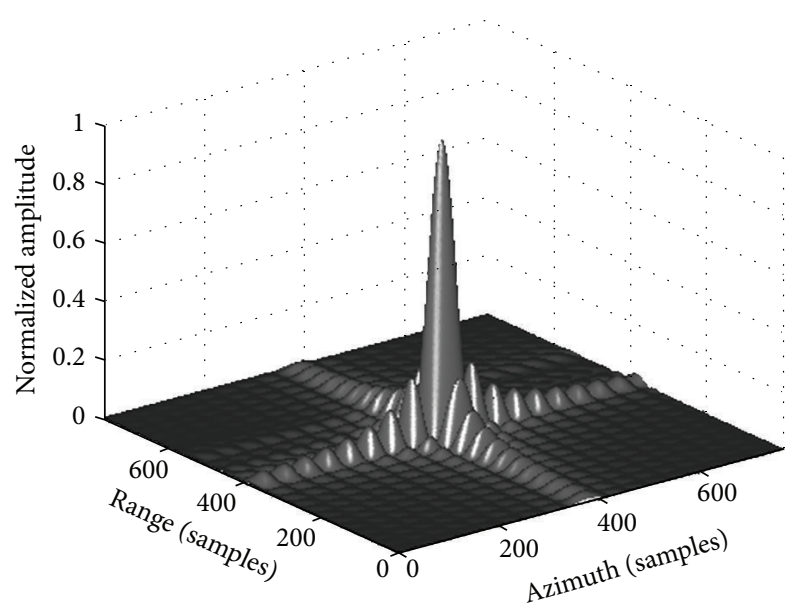

(a) 2D impulse response

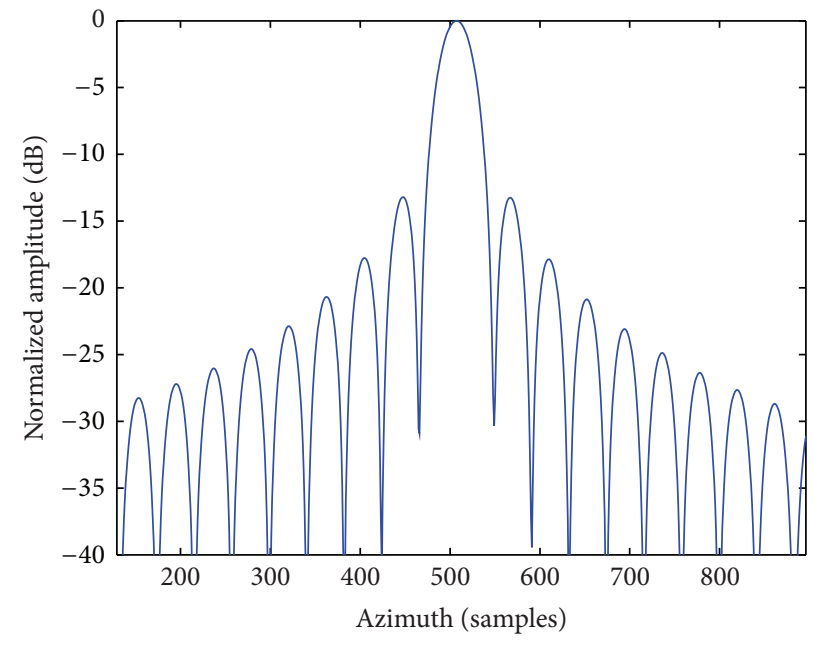

(b) Azimuth impulse response

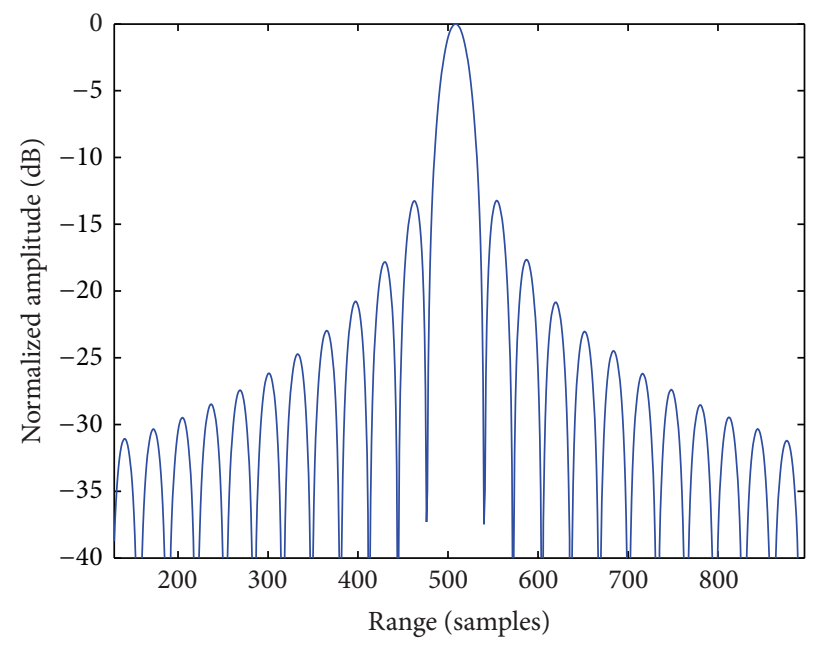

(c) Range impulse response

FIGURE 4: Fringe point results using the proposed range model.

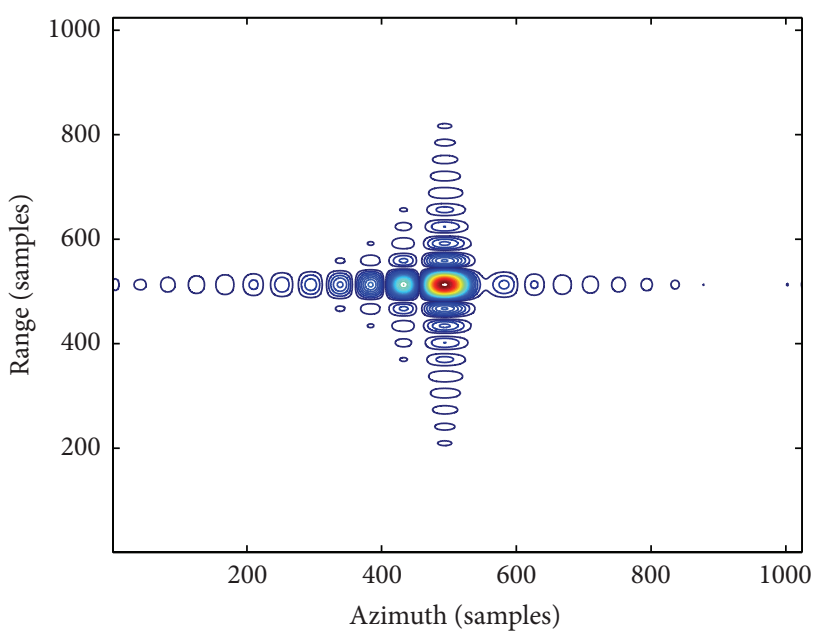

(a) Quadratic range model

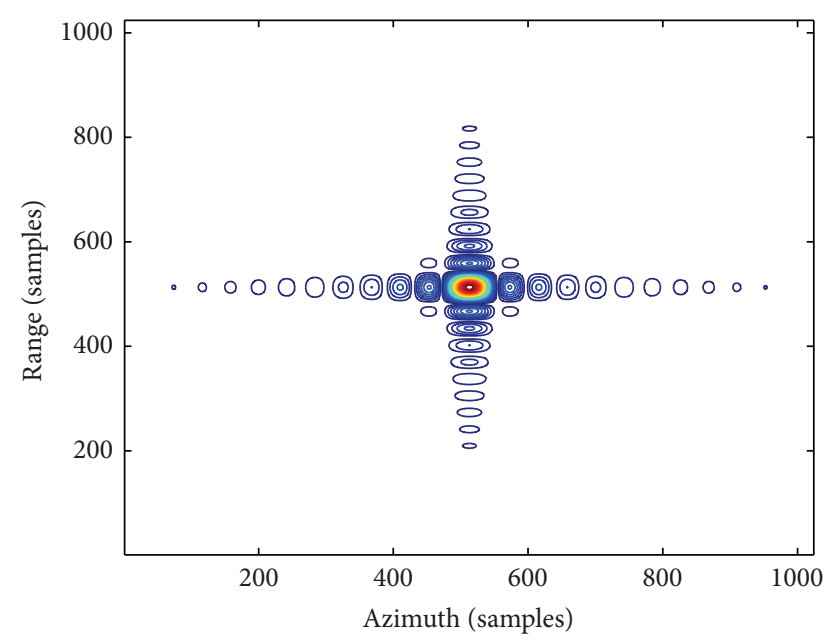

(b) Proposed range model

FIGURE 5: Contour plots comparison using different range models. 


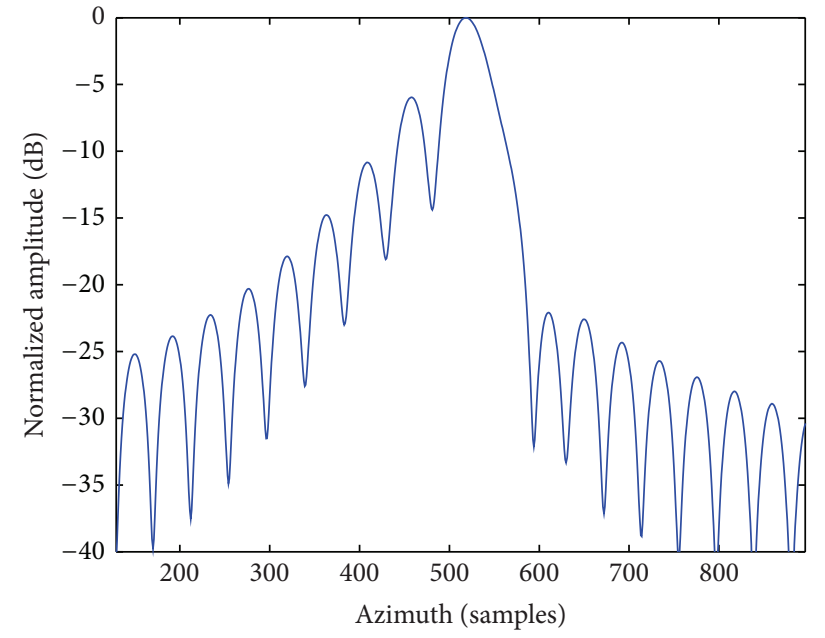

(a) Quadratic range model

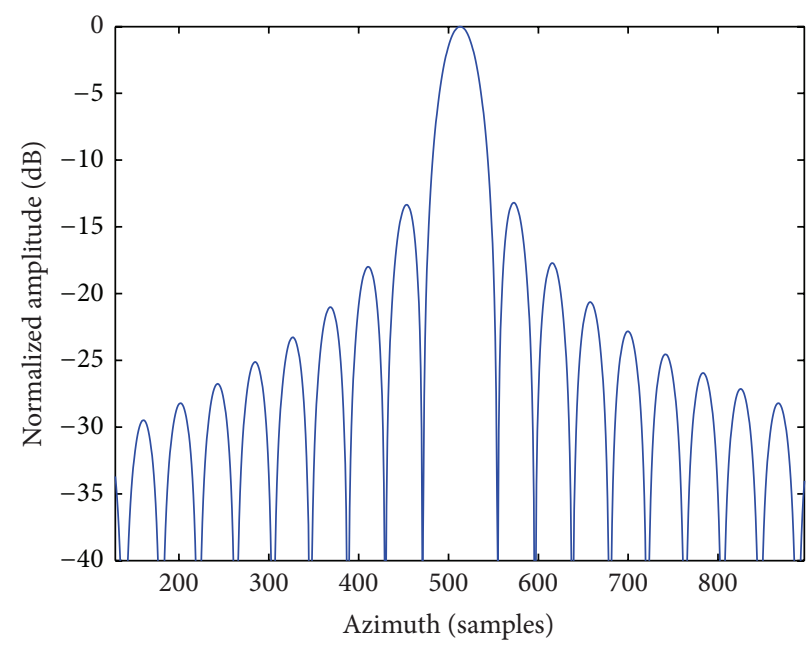

(b) Proposed range model

FIGURE 6: Azimuth impulse responses comparison using different range models.

azimuth match filtering function, which can be obtained as follows:

$$
H_{2}\left(t_{m}, f_{a}\right)=\exp \left[-j\left(\Phi_{a}\left(f_{a}\right)\right)\right] .
$$

(5) Perform azimuth IFFT to obtain a well-focused SAR image.

\section{Simulations and Results}

In this section, several examples are provided to illustrate the performance of the proposed method. Because of the complex configuration, the high-order terms in the slant range cannot be ignored. If inappropriately approximated, the imaging focusing resolution may be affected significantly. Some simulations are conducted to compare the influences of keeping the terms up to the quadratic term (quadratic range model) and the higher order term (proposed range model). Parameters used in the simulations are listed in Table 1. The imaging results of center point and fringe point in the imaging area using the proposed range model are given in Figures 3 and 4 . In order to confirm the necessity and validity using the higher order range model in this special configuration, some simulations are also conducted to compare the differences using the quadratic range model and the proposed range model, and the results are shown in Figures 5 and 6 (here, the center point is selected in comparison).

Figure 3 depicts the results of the center point using the proposed range model. Figure 3(a) is the 2D impulse response; Figures 3(b) and 3(c) are the azimuth impulse response and the range impulse response, respectively. The 2D impulse response of the fringe target point using the proposed range model is shown in Figure 4(a), and the azimuth impulse response and the range impulse response are given in Figures 4(b) and 4(c), respectively. We can see from Figures 3 and 4 that both center point and fringe point are well focused in range direction and azimuth direction. For the complexity of the MBFL-SAR configuration, if the slant range is not approximated accurately enough, the phase term in the 2D frequency spectrum will not be compensated completely in the azimuth direction. It is easy to find that the image of the point target suffers from distortion and main-lobe broadening when the slant range keeps the terms up to the quadratic term in Figures 5(a) and 6(a). On the other hand, if we are using the proposed range model, the imaging focusing performance can be obviously improved, as given in Figures 5(b) and 6(b). It is obvious that the image is well focused in the azimuth direction, which confirms it necessary to approximate appropriately in the MBFL-SAR configuration.

Table 2 lists the image quality parameters of the center point and fringe point using the proposed method to further illustrate the validity of the proposed algorithm. PSLR and ISLR represent peak side-lobe ratio and integrated side-lobe ratio, respectively. Besides, the theoretical PSLR and ISLR are $-13.26 \mathrm{~dB}$ and $-9.8 \mathrm{~dB}$, respectively. We can see that the image quality parameters of both the center point and fringe point using our proposed method are all close to the theoretical ones, which indicate satisfactory imaging results and further validate the effectiveness and feasibility.

\section{Conclusions}

In this paper, we developed an imaging algorithm for MBFLSAR configuration, a special MIMO radar mode. The MBFLSAR imaging geometric model is established and the signal model is analyzed firstly. The high-order term in the slant range cannot be neglected because of the presence of high velocity and acceleration and the addition of two squareroot terms. Also, the difficulty in direct-using the POSP in this configuration is discussed. Then $2 \mathrm{D}$ frequency spectrum is deduced using the method of series reversion based on the appropriate approximation of the slant range. Based on the derived spectrum, the corresponding imaging method is 
developed. The high-order term introduced from this configuration is compensated, together with range compression, at the beginning of the algorithm. RCMC is finished in the range time azimuth frequency domain as the range-variant $k$ coefficients. The focused SAR image can be obtained through the azimuth compression. The feasibility and efficiency of the proposed approach are validated with the simulation experiments.

\section{Conflict of Interests}

The authors declare that there is no conflict of interests regarding the publication of this paper.

\section{Acknowledgments}

This work was supported by the National Natural Science Foundation of China under Grants 61001211 and 61303035, by the Fundamental Research Funds for the Central Universities (K5051202016), and by the Science Foundation for Navigation (20110181004).

\section{References}

[1] I. G. Cumming and F. H. Wong, Digital Processing of Synthetic Aperture Radar Data: Algorithms and Implementation, Artech House, Norwood, Mass, USA, 2005.

[2] D. L. Mensa, High Resolution Radar Cross-Section Imaging, Artech House, Boston, Mass, USA, 1991.

[3] S. A. Hovanessian, Introduction to Synthetic Array and Imaging Radar, Artech House, Norwood, Mass, USA, 1980.

[4] R. Kapoor, A. Banerjee, G. A. Tsihrintzis, and N. Nandhakumar, "UWB radar detection of targets in foliage using alpha-stable clutter models," IEEE Transactions on Aerospace and Electronic Systems, vol. 35, no. 3, pp. 819-834, 1999.

[5] G. Wang, X. Xia, and V. C. Chen, "Radar imaging of moving targets in foliage using multifrequency multiaperture polarimetric SAR," IEEE Transactions on Geoscience and Remote Sensing, vol. 41, no. 8, pp. 1755-1764, 2003.

[6] E. Fishler, A. Haimovich, R. Blum, D. Chizhik, L. Cimini, and R. Valenzuela, "MIMO radar: An idea whose time has come," in Proceedings of the IEEE Radar Conference, pp. 71-78, April 2004.

[7] E. Fishler, A. Haimovich, R. Blum, L. Cimini, D. Chizhik, and R. Valenzuela, "Performance of MIMO radar systems: advantages of angular diversity," in Proceedings of the 38th Asilomar Conference on Signals, Systems and Computers, pp. 305-309, November 2004.

[8] R. Sharma, "Analysis of MIMO radar ambiguity functions and implications on clear region," in Proceedings of the IEEE International Radar Conference (RADAR '10), pp. 544-548, Washington, DC, USA, May 2010.

[9] J. Li and P. Stoica, MIMO Radar Signal Processing, Wiley, New York, NY, USA, 2009.

[10] W. Roberts, P. Stoica, J. Li, T. Yardibi, and F. A. Sadjadi, "Iterative adaptive approaches to MIMO radar imaging," IEEE Journal on Selected Topics in Signal Processing, vol. 4, no. 1, pp. 5-20, 2010.

[11] J. Li, G. Liao, and H. Griffiths, "Bistatic MIMO radar spacetime adaptive processing," in Proceedings of the IEEE Radar Conference: in the Eye of the Storm (RadarCon '11), pp. 498-501, Kansas, Mo, USA, May 2011.
[12] D. W. Bliss and K. W. Forsythe, "Multiple-input multiple-output (MIMO) radar and imaging: degrees of freedom and resolution," in Proceedings of the 37th Asilomar Conference on Signals, Systems and Computers, pp. 54-59, November 2003.

[13] I. Walterscheid, T. Espeter, J. Klare, A. R. Brenner, and J. H. G. Ender, "Potential and limitations of forward-looking bistatic SAR," in Proceedings of the 30th IEEE International Geoscience and Remote Sensing Symposium (IGARSS '10), pp. 216-219, July 2010.

[14] J. Wu, J. Yang, Y. Huang, H. Yang, and H. Wang, "Bistatic forward-looking SAR: theory and challenges," in Proceedings of the IEEE Radar Conference (RADAR '09), pp. 1-4, Pasadena, Calif, USA, May 2009.

[15] Z. Shi, H. Wang, P. Zhang, X. Tang, B. Wu, and C. Wang, "Study on the probability of successful handoff of missile trajectory from midcourse guidance to terminal guidance," in Proceedings of the International Conference on Computational and Information Sciences (ICCIS '10), pp. 1005-1008, December 2010.

[16] Q. Zhao, X. Zhang, W. Song, and F. Lu, "Rotational analysis of certain terminal-guidance projectile," in Proceedings of the 8th International Conference on Electronic Measurement and Instruments (ICEMI '07), pp. 4415-4418, August 2007.

[17] C. Wu, "A digital system to produce imagery from SAR data," in Proceedings of AIAA Conference on Systems Design Driven by Sensors, October 1976.

[18] J. R. Bennett and I. G. Cumming, "A digital processor for the production of SEASAT synthetic aperture radar imagery", in Proceedings of Symposium on Machine Processing of Remotely Sensed Data, June 1979.

[19] C. Wu, K. Y. Liu, and M. Y. Jin, "Modeling and a correlation algorithm for spaceborne SAR signals," IEEE Transactions on Aerospace and Electronic Systems, vol. 18, no. 5, pp. 563-575, 1982.

[20] R. K. Raney, H. Runge, R. Bamler, I. G. Cumming, and F. H. Wong, "Precision SAR processing using chirp scaling," IEEE Transactions on Geoscience and Remote Sensing, vol. 32, no. 4, pp. 786-799, 1994.

[21] M. Soumekh, "Bistatic synthetic aperture radar inversion with application in dynamic object imaging," IEEE Transactions on Signal Processing, vol. 39, no. 9, pp. 2044-2055, 1991.

[22] M. Soumekh, "Wide-bandwidth continuous-wave monostatic/bistatic synthetic aperture radar imaging," in Proceedings of IEEE International Conference on Image Processing (ICIP '98), vol. 3, pp. 361-365, IEEE, Chicago, Ill, USA, October 1998.

[23] M. Soumekh, J. Choi, and W. Zwolinski, "Target imaging using a synthesized bistatic radar," in Proceedings of the IEEE International Conference on Systems Engineering, pp. 39-42, August 1989.

[24] Y. L. Neo, F. H. Wong, and I. G. Cumming, "Focusing bistatic SAR images using non-linear chirp scaling," in Proceedings of International Conference on Radar Systems, 2004.

[25] G. W. Davidson, I. G. Cumming, and M. R. Ito, "A chirp scaling approach for processing squint mode SAR data," IEEE Transactions on Aerospace and Electronic Systems, vol. 32, no. 1, pp. 121-133, 1996.

[26] D. D' Aria, A. M. Guarnieri, and F. Rocca, "Focusing bistatic synthetic aperture radar using dip move out," IEEE Transactions on Geoscience and Remote Sensing, vol. 42, no. 7, pp. 1362-1376, 2004.

[27] A. M. Guarnieri and F. Rocca, "Reduction to monostatic focusing of bistatic or motion uncompensated SAR surveys," IEE 
Proceedings: Radar, Sonar and Navigation, vol. 153, no. 3, pp. 199-207, 2006.

[28] Y. L. Neo, F. Wong, and I. G. Cumming, "A two-dimensional spectrum for bistatic SAR processing using series reversion," IEEE Geoscience and Remote Sensing Letters, vol. 4, no. 1, pp. 93-96, 2007.

[29] Y. L. Neo, F. H. Wong, and I. G. Cumming, "Processing of azimuth-invariant bistatic SAR data using the range doppler algorithm," IEEE Transactions on Geoscience and Remote Sensing, vol. 46, no. 1, pp. 14-21, 2008. 

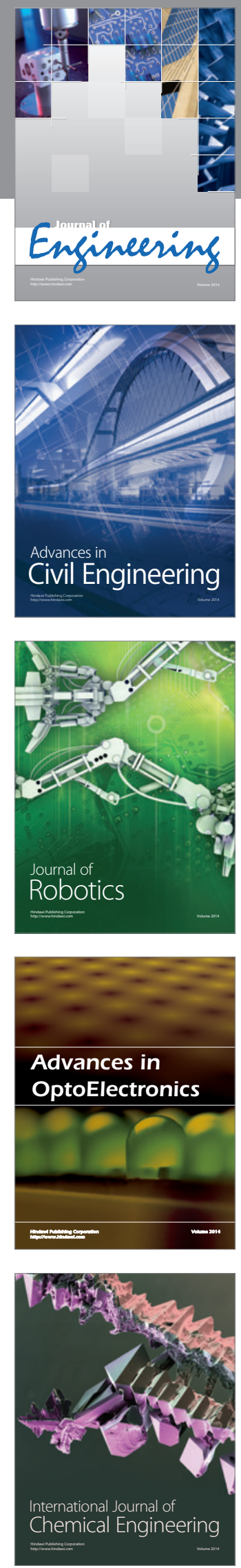

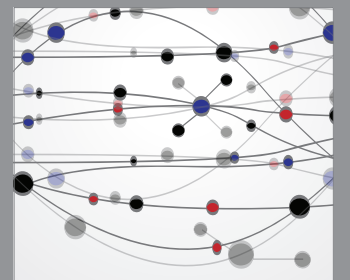

The Scientific World Journal
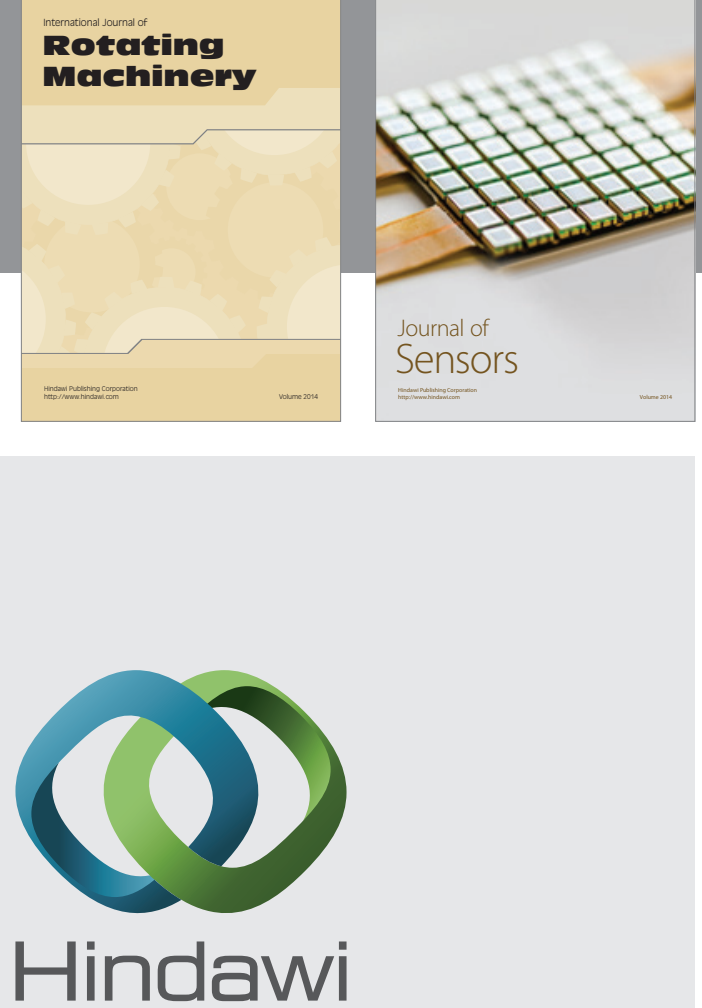

Submit your manuscripts at http://www.hindawi.com
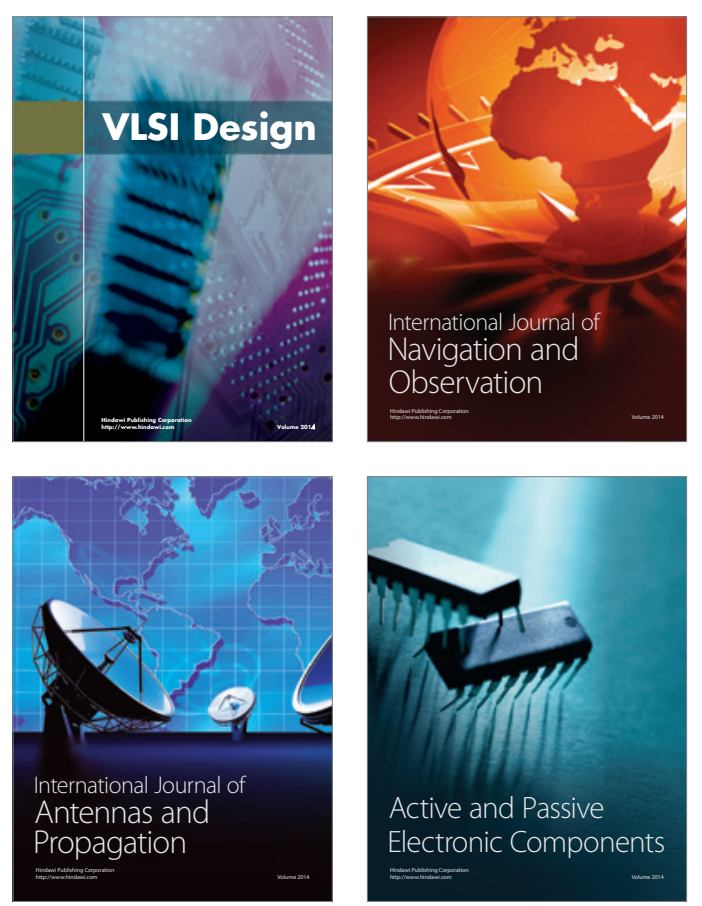
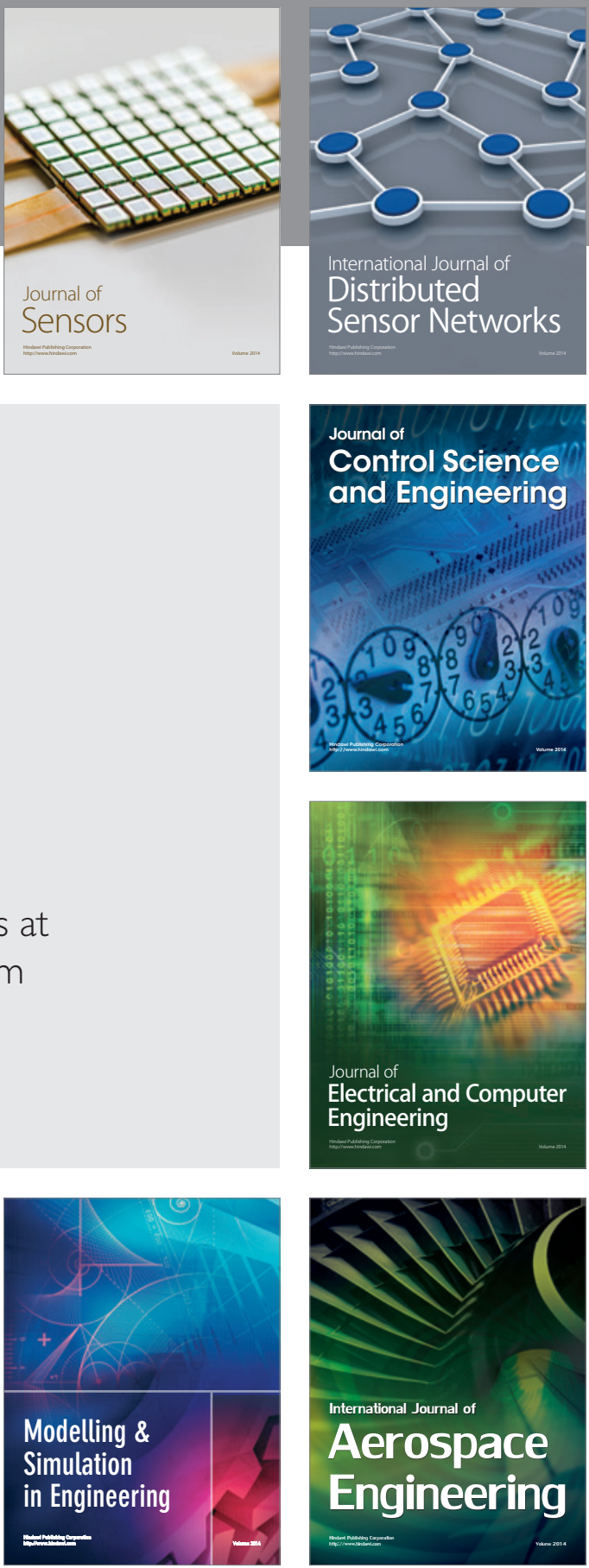

Journal of

Control Science

and Engineering
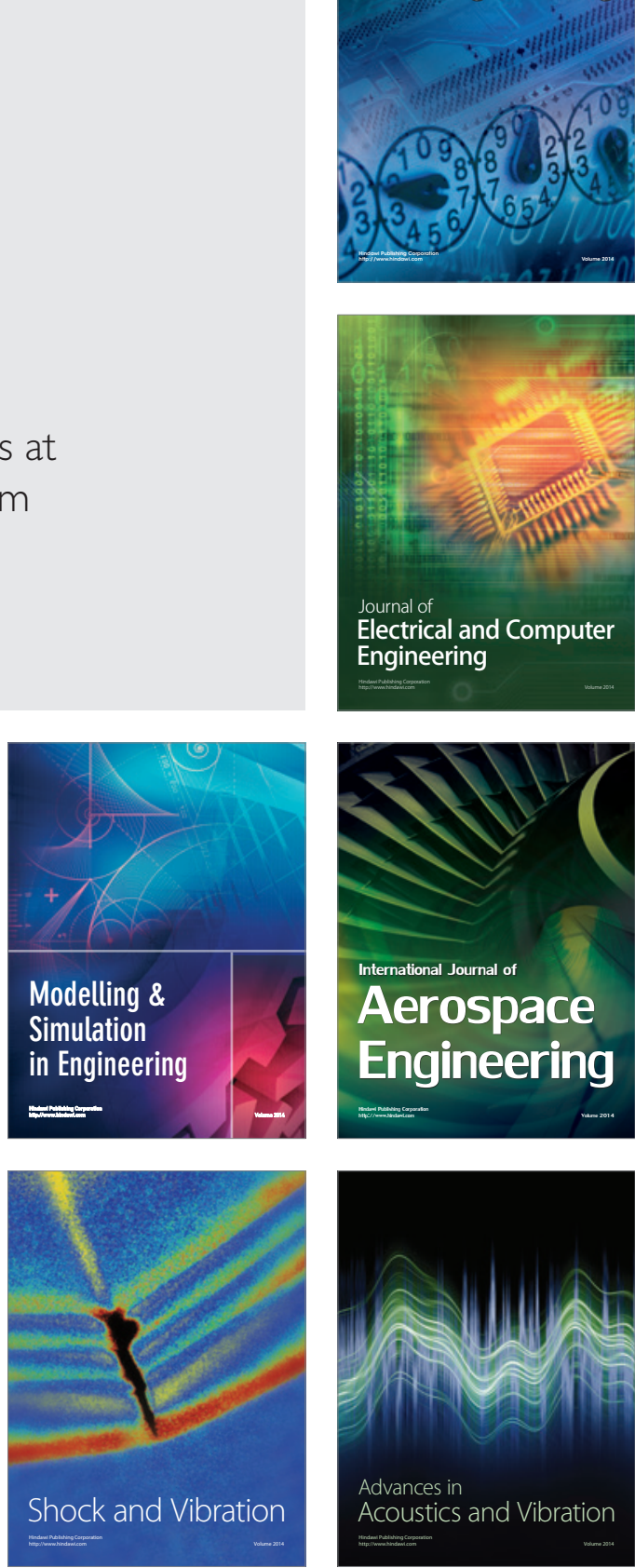